Homology, Homotopy and Applications, vol. 18(2), 2016, pp.71-84

\title{
ON THE COHOMOLOGY OF ORIENTED GRASSMANN MANIFOLDS
}

\author{
JÚLIUS KORBAŠ AND TOMÁŠ RUSIN
}

(communicated by Donald M. Davis)

\begin{abstract}
This paper presents a new approach to studying the kernel of the additive homomorphism from $H^{q}\left(G_{n, k}\right)$ to $H^{q+1}\left(G_{n, k}\right)$ given by the cup-product with the first Stiefel-Whitney class of the canonical $k$-plane bundle over the Grassmann manifold $G_{n, k}$ of all $k$-dimensional vector subspaces in Euclidean $n$-space. This method enables us to improve the understanding of the $\mathbb{Z}_{2}$-cohomology of the "oriented" Grassmann manifold $\widetilde{G}_{n, k}$ of oriented $k$-dimensional vector subspaces in Euclidean $n$-space. In particular, we derive new information on the characteristic rank of the canonical oriented $k$-plane bundle over $\widetilde{G}_{n, k}$ and the $\mathbb{Z}_{2}$-cup-length of $\widetilde{G}_{n, k}$. Our results on the cup-length for three infinite families of the manifolds $\widetilde{G}_{n, 3}$ confirm the corresponding claims of Fukaya's conjecture from 2008.
\end{abstract}

Dedicated to Professor Ulrich Koschorke on the occasion of his 75-th birthday.

\section{Introduction}

The $\mathbb{Z}_{2}$-cohomology algebra of the "unoriented" Grassmann manifold $G_{n, k}$ ( $k \leqslant$ $n-k$ ) of $k$-dimensional vector subspaces in $\mathbb{R}^{n}$ has a simple description in terms of generators and relations $[\mathbf{3}]$ : we can write

$$
H^{*}\left(G_{n, k}\right)=\mathbb{Z}_{2}\left[w_{1}, \ldots, w_{k}\right] / I_{n, k},
$$

where $\operatorname{dim}\left(w_{i}\right)=i$ and the ideal $I_{n, k}$ is generated by the $k$ homogeneous components of $\left(1+w_{1}+\cdots+w_{k}\right)^{-1}$ in dimensions $n-k+1, \ldots, n$. If $\gamma_{n, k}$ (briefly $\gamma$ ) denotes the canonical $k$-plane bundle over $G_{n, k}$, then the indeterminate $w_{i}$ is a representative of the $i$ th Stiefel-Whitney class $w_{i}(\gamma)$ in the quotient algebra $H^{*}\left(G_{n, k}\right)$. For $w_{i}(\gamma)$, we shall also use $w_{i}$ as an abbreviation. Note that all cohomology in this paper will be taken with coefficients in $\mathbb{Z}_{2}$. Also note that $w_{i}=w_{i}(\gamma)$ should not be confused with the Stiefel-Whitney classes of the manifold, namely $w_{i}\left(G_{n, k}\right)=w_{i}\left(T G_{n, k}\right)$, the Stiefel-Whitney classes of its tangent bundle.

The authors were supported in part by two grants of VEGA (Slovakia). J. Korbaš was also partially affiliated with the Mathematical Institute, Slovak Academy of Sciences, Bratislava.

Received May 19, 2015, revised January 11, 2016, January 17, 2016; published on July 6, 2016. 2010 Mathematics Subject Classification: 57R20, 55R25.

Key words and phrases: Stiefel-Whitney class, characteristic rank, cup-length, Grassmann manifold. Article available at http://dx.doi.org/10.4310/HHA.2016.v18.n2.a4

Copyright (C) 2016, International Press. Permission to copy for private use granted. 
Besides $\gamma_{n, k}$ and its $(n-k)$-dimensional orthogonal complement $\gamma_{n, k}^{\perp}$ (briefly $\gamma^{\perp}$ ), over $G_{n, k}$ we have a nontrivial line bundle $\xi=\operatorname{det}(\gamma)=\operatorname{det}\left(\gamma^{\perp}\right)$. Then the "oriented" Grassmann manifold $\widetilde{G}_{n, k}$ of oriented $k$-dimensional vector subspaces in $\mathbb{R}^{n}$ which, of course, is a double cover of $G_{n, k}$, can be interpreted as the 0 -sphere bundle of $\xi$ ([9, Corollary 12.3] or [12, Theorem 5.7.11]), to which one has an exact sequence of Gysin type,

$$
\stackrel{\psi}{\longrightarrow} H^{j-1}\left(G_{n, k}\right) \stackrel{w_{1}}{\longrightarrow} H^{j}\left(G_{n, k}\right) \stackrel{p^{*}}{\longrightarrow} H^{j}\left(\widetilde{G}_{n, k}\right) \stackrel{\psi}{\longrightarrow} H^{j}\left(G_{n, k}\right) \stackrel{w_{1}}{\longrightarrow} .
$$

We write here and elsewhere $H^{j-1}\left(G_{n, k}\right) \stackrel{w_{1}}{\longrightarrow} H^{j}\left(G_{n, k}\right)$ for the homomorphism given by the cup-product with the Stiefel-Whitney class $w_{1}(\xi)=w_{1}=w_{1}\left(\gamma^{\perp}\right)$, and $p: \widetilde{G}_{n, k} \rightarrow G_{n, k}$ is the obvious covering projection. Note that $\widetilde{G}_{n, k}$ is always orientable as a manifold, whereas $G_{n, k}$ is an orientable manifold if and only if $n$ is even.

It is known [14] that $\operatorname{Im}\left(p^{*}: H^{*}\left(G_{n, k}\right) \longrightarrow H^{*}\left(\widetilde{G}_{n, k}\right)\right)$ is a self-annihilating subspace of $H^{*}\left(\widetilde{G}_{n, k}\right)$ of half the dimension. Very little is known about the algebra $H^{*}\left(\widetilde{G}_{n, k}\right)$, apart from the cases of $(n-1)$-dimensional spheres $\widetilde{G}_{n, 1} \cong S^{n-1}$ and complex quadrics $\widetilde{G}_{n, 2}$. This is due to the fact that it is difficult to obtain information on cohomology classes that generate

$$
\operatorname{Im}(\psi) \cong H^{*}\left(\widetilde{G}_{n, k}\right) / \operatorname{Ker}(\psi)=H^{*}\left(\widetilde{G}_{n, k}\right) / \operatorname{Im}\left(p^{*}\right)
$$

A reason for this is that, in general, it is hard to calculate explicitly in $H^{*}\left(G_{n, k}\right)$ and determine the kernel of $w_{1}$; of course, by (2), the latter vector space is the same as $\operatorname{Im}(\psi)$.

Over $\widetilde{G}_{n, k}$ we have the canonical oriented $k$-plane bundle $\widetilde{\gamma}_{n, k}$ (briefly $\widetilde{\gamma}$ ), which is isomorphic to $p^{*}(\gamma)$. As a consequence, $p^{*}\left(w_{i}\right)=\widetilde{w}_{i}$ for all $i$, where $\widetilde{w}_{i}$ is an abbreviation, used throughout the paper, for the Stiefel-Whitney class $w_{i}\left(\widetilde{\gamma}_{n, k}\right)$; note $\widetilde{w}_{1}=0$. The subspace $C(j ; n, k):=\operatorname{Im}\left(p^{*}\right)$ of the $\mathbb{Z}_{2}$-vector space $H^{j}\left(\widetilde{G}_{n, k}\right)$ is the characteristic subspace (all its elements can be expressed in the Stiefel-Whitney characteristic classes of $\widetilde{\gamma}_{n, k}$; that is why we call it by this name). If we $\operatorname{denote} \operatorname{dim}(C(j ; n, k))$ by $\chi_{j}\left(\widetilde{G}_{n, k}\right)$ and $\operatorname{dim}(\operatorname{Im}(\psi))$ by $\alpha_{j}\left(\widetilde{G}_{n, k}\right)$, then (see $\left.(3)\right)$

$$
\chi_{j}\left(\widetilde{G}_{n, k}\right)+\alpha_{j}\left(\widetilde{G}_{n, k}\right)=b_{j}\left(\widetilde{G}_{n, k}\right),
$$

the right-hand side being the $j$ th $\mathbb{Z}_{2}$-Betti number of $\widetilde{G}_{n, k}$.

Recall $[\mathbf{1 0}, \mathbf{8}]$ that, for a real vector bundle $\alpha$ over a path-connected $C W$-complex $X$, its characteristic rank, $\operatorname{charrank}(\alpha)$, is defined to be the greatest integer $q, 0 \leqslant$ $q \leqslant \operatorname{dim}(X)$, such that every cohomology class in $H^{j}(X), 0 \leqslant j \leqslant q$, is a polynomial in the Stiefel-Whitney classes $w_{i}(\alpha) \in H^{i}(X)$. In particular (see [7]), if $T M$ is the tangent bundle of a smooth closed connected manifold $M$, then charrank $(T M)$ is the characteristic rank of $M$, denoted charrank $(M)$.

Now the greatest integer $q$ such that

$$
\alpha_{0}\left(\widetilde{G}_{n, k}\right)=\alpha_{1}\left(\widetilde{G}_{n, k}\right)=\cdots=\alpha_{q}\left(\widetilde{G}_{n, k}\right)=0
$$

is nothing but the characteristic rank of $\widetilde{\gamma}_{n, k}$, briefly charrank $\left(\widetilde{\gamma}_{n, k}\right)$.

Of course, we have

$$
\alpha_{1+\operatorname{charrank}\left(\widetilde{\gamma}_{n, k}\right)}\left(\widetilde{G}_{n, k}\right) \neq 0,
$$

since $1+\operatorname{charrank}\left(\widetilde{\gamma}_{n, k}\right)$ is the least degree, in which an "anomalous" (not expressible 
exclusively in the Stiefel-Whitney classes of $\left.\widetilde{\gamma}_{n, k}\right)$ generator of $H^{*}\left(\widetilde{G}_{n, k}\right)$ appears. Note that (see $(2)) \operatorname{charrank}\left(\widetilde{\gamma}_{n, k}\right) \geqslant j$ (for some $j$ ) if and only if $p^{*}: H^{i}\left(G_{n, k}\right) \longrightarrow$ $H^{i}\left(\widetilde{G}_{n, k}\right)$ is surjective or, equivalently, $w_{1}: H^{i}\left(G_{n, k}\right) \rightarrow H^{i+1}\left(G_{n, k}\right)$ is injective, for all non-negative integers $i \leqslant j$.

When we know that some cohomology group of $\widetilde{G}_{n, k}$, in a degree not exceeding half of $\operatorname{dim}\left(\widetilde{G}_{n, k}\right)$, does not vanish, we can use it to obtain an upper bound for $\operatorname{charrank}\left(\widetilde{\gamma}_{n, k}\right)$. More precisely, due to the fact that the subspace $\operatorname{Im}\left(p^{*}\right) \subset H^{*}\left(\widetilde{G}_{n, k}\right)$ is self-annihilating, one can easily adjust the proof of $[\mathbf{2}$, Theorem 2.1$]$ to verify that if $H^{t}\left(\widetilde{G}_{n, k}\right) \neq 0$ for some $t \leqslant \frac{1}{2} k(n-k)$, then we have

$$
\operatorname{charrank}\left(\widetilde{\gamma}_{n, k}\right) \leqslant k(n-k)-t-1 \text {. }
$$

Under certain conditions, the characteristic rank of a vector bundle over a smooth closed connected manifold $M$ and the $\mathbb{Z}_{2}$-cup-length, denoted by $\operatorname{cup}(M)$, are nicely related, as shown in the following generalization of [7, Theorem 1.1] which, in particular, will be used (in Section 3) for deriving upper bounds or exact values for the cup-length of $\widetilde{G}_{n, k}$.

Theorem 1.1 (Naolekar and Thakur [10]). Let $M$ be a connected closed smooth $d$ dimensional manifold. Let $\alpha$ be a vector bundle over $M$ satisfying the following: there exists $j, j \leqslant \operatorname{charrank}(\alpha)$, such that every monomial $w_{i_{1}}(\alpha) \cdots w_{i_{s}}(\alpha), 0 \leqslant i_{t} \leqslant j$, in dimension $d$ vanishes. Then

$$
\operatorname{cup}(M) \leqslant 1+\frac{d-j-1}{r_{M}}
$$

where $r_{M}$ is the smallest positive integer such that $\widetilde{H}^{r_{M}}(M) \neq 0$.

In addition, for any $j \leqslant \operatorname{charrank}\left(\widetilde{\gamma}_{n, k}\right)$, one sees that both the $w_{1}$-homomorphisms in the Gysin sequence (2) are injective and the homomorphism $p^{*}: H^{j}\left(G_{n, k}\right) \longrightarrow$ $H^{j}\left(\widetilde{G}_{n, k}\right)$ is surjective, thus we have

$$
H^{j}\left(\widetilde{G}_{n, k}\right) \cong H^{j}\left(G_{n, k}\right) / \operatorname{Im}\left(w_{1}: H^{j-1}\left(G_{n, k}\right) \rightarrow H^{j}\left(G_{n, k}\right)\right) .
$$

Of course, now $\operatorname{dim}\left(\operatorname{Im}\left(w_{1}: H^{j-1}\left(G_{n, k}\right) \rightarrow H^{j}\left(G_{n, k}\right)\right)\right)=b_{j-1}\left(G_{n, k}\right)$. Consequently, if $j \leqslant \operatorname{charrank}\left(\widetilde{\gamma}_{n, k}\right)$, then we have for the Betti number $b_{j}\left(\widetilde{G}_{n, k}\right)$ that

$$
b_{j}\left(\widetilde{G}_{n, k}\right)=b_{j}\left(G_{n, k}\right)-b_{j-1}\left(G_{n, k}\right) .
$$

The difference of the $\mathbb{Z}_{2}$-Betti numbers of the Grassmann manifold $G_{n, k}$ on the right-hand side is readily calculable from the Poincaré polynomial, and is nothing but the number of linearly independent semi-invariants of degree $k$ and weight $j$ of a binary form of degree $n-k$, provided $j \leqslant \frac{k(n-k)}{2}$ (note that the latter number equals $\frac{1}{2} \operatorname{dim}\left(G_{n, k}\right)$ ), by a theorem of Cayley and Sylvester (see [11, Satz 2.21]). This interesting interpretation of the Betti numbers $b_{j}\left(\widetilde{G}_{n, k}\right)$ for

$$
j \leqslant \min \left\{\operatorname{charrank}\left(\widetilde{\gamma}_{n, k}\right), \frac{k(n-k)}{2}\right\}
$$

seems to have remained unnoticed thus far.

Theorem 2.1 in [8], on lower bounds or exact values for charrank $\left(\widetilde{\gamma}_{n, k}\right)(3 \leqslant k \leqslant$ $n-k)$, gives information on the structure of the $\mathbb{Z}_{2}$-cohomology of the manifold $\widetilde{G}_{n, k}$. 
In the present paper, we add further results. As compared to [8], we present a different approach to studying the kernel of $w_{1}$. Some of the new results on the characteristic rank presented here imply new exact values of the $\mathbb{Z}_{2}$-cup-length of $\widetilde{G}_{n, k}$. In particular, our results on the cup-length of three infinite families of the manifolds $\widetilde{G}_{n, 3}$ in Theorem 3.6(2) confirm the corresponding claims of Fukaya's conjecture [4, p. 196].

\section{An approach to studying the kernel of $w_{1}$}

The aim of this section is to develop tools for studying the kernel of the homomorphism $w_{1}: H^{j}\left(G_{n, k}\right) \longrightarrow H^{j+1}\left(G_{n, k}\right)$.

A key rôle will be played by the fact that, for the $\mathbb{Z}_{2}$-vector space $H^{j}\left(G_{n, k}\right)(k \leqslant$ $n-k)$, the set

$$
\left\{w_{1}^{a_{1}} \cdots w_{k}^{a_{k}} ; \sum_{i=1}^{k} i a_{i}=j, \sum_{i=1}^{k} a_{i} \leqslant n-k\right\}
$$

is an additive basis. This follows from [9, Corollary 6.7]; another proof can be found in [5]. We shall refer to the basis (4) as "standard basis" in this paper. We say that an element, $w_{1}^{a_{1}} \cdots w_{k}^{a_{k}} \in H^{j}\left(G_{n, k}\right)$, of the standard basis is regular (with respect to the homomorphism $\left.w_{1}: H^{j}\left(G_{n, k}\right) \longrightarrow H^{j+1}\left(G_{n, k}\right)\right)$ if its $w_{1}$-image is an element of the standard basis for $H^{j+1}\left(G_{n, k}\right)$, that is, if $\sum_{i=1}^{k} a_{i}<n-k$. An element of the standard basis that is not regular is said to be singular.

Of course,

$$
\operatorname{dim}\left(\operatorname{Im}\left(w_{1}: H^{j}\left(G_{n, k}\right) \longrightarrow H^{j+1}\left(G_{n, k}\right)\right)\right)
$$

is greater than or equal to the number of regular elements of the standard basis for $H^{j}\left(G_{n, k}\right)$ and

$$
\operatorname{dim}\left(\operatorname{Ker}\left(w_{1}: H^{j}\left(G_{n, k}\right) \longrightarrow H^{j+1}\left(G_{n, k}\right)\right)\right)=\alpha_{j}\left(\widetilde{G}_{n, k}\right)
$$

does not exceed the number of singular elements of the standard basis for $H^{j}\left(G_{n, k}\right)$. The latter inequality can be concretized. Indeed, let $p(\{1,2, \ldots, k-1\}, x)$ denote the number of partitions of a non-negative integer $x$ into parts, each taken from the set $\{1,2, \ldots, k-1\}$; in particular, if $x \leqslant k-1$, then $p(\{1,2, \ldots, k-1\}, x)=p(x)$ is the total number of partitions of $x$.

Proposition 2.1. For the Grassmann manifold $G_{n, k}(2 \leqslant k \leqslant n-k)$, we have the following:

(a) If $1 \leqslant x \leqslant n-k$, then all the elements in the standard basis for $H^{n-k-x}\left(G_{n, k}\right)$ are regular, thus we have $\alpha_{n-k-x}\left(\widetilde{G}_{n, k}\right)=0$.

(b) If $x \geqslant 0$, then precisely $p(\{1,2, \ldots, k-1\}, x)$ elements of the standard basis for $H^{n-k+x}\left(G_{n, k}\right)$ are singular; thus $\alpha_{n-k+x}\left(\widetilde{G}_{n, k}\right) \leqslant p(\{1,2, \ldots, k-1\}, x)$.

Proof. Part (a). Let $w_{1}^{a_{1}} \cdots w_{k}^{a_{k}}$ be an element of the standard basis in $H^{n-k-x}\left(G_{n, k}\right)$. We have

$$
\sum_{i=1}^{k} a_{i} \leqslant \sum_{i=1}^{k} i a_{i}=n-k-x
$$

thus the equality $\sum_{i=1}^{k} a_{i}=n-k$ is impossible; in other words, each basis element 
is now regular.

Part (b). If $w_{1}^{a_{1}} \cdots w_{k}^{a_{k}}$ is a singular element of the standard basis in $H^{n-k+x}\left(G_{n, k}\right)$, then clearly $a_{1}+a_{2}+\cdots+a_{k}=n-k$, whence

$$
a_{2}+2 a_{3}+\cdots+(k-1) a_{k}=x .
$$

Since $a_{1}$ is uniquely determined by the equation $a_{1}=n-k-a_{2}-\cdots-a_{k}$, the number of singular elements of the standard basis is equal to $p(\{1,2, \ldots, k-1\}, x)$.

Remark 2.2. Of course, the vanishing of $\alpha_{n-k-x}\left(\widetilde{G}_{n, k}\right)$ for all $x$ in Proposition 2.1(a) is equivalent to the known inequality $[8,(2.5)] \operatorname{charrank}\left(\widetilde{\gamma}_{n, k}\right) \geqslant n-k-1$.

The next lemma is elementary and stated without proof.

Lemma 2.3. Let $\vec{a}_{1}, \ldots, \vec{a}_{m}, \vec{b}_{1}, \ldots, \vec{b}_{n}$ be linearly independent vectors in a vector space $V$ over a field $K$. If $\vec{c}_{1}, \ldots, \vec{c}_{s}(s \leqslant n)$ are linearly independent vectors in the linear span $\left[\vec{b}_{1}, \ldots, \vec{b}_{n}\right] \subset V$, then also the vectors $\vec{a}_{1}, \ldots, \vec{a}_{m}, \vec{c}_{1}, \ldots, \vec{c}_{s}$ are linearly independent.

In $\mathbb{Z}_{2}\left[w_{1}, \ldots, w_{k}\right]$, let $\bar{w}_{i}\left(w_{1}, \ldots, w_{k}\right)$ (briefly $\left.\bar{w}_{i}\right)$ denote the homogeneous component of $\left(1+w_{1}+\cdots+w_{k}\right)^{-1}=1+w_{1}+\cdots+w_{k}+\left(w_{1}+\cdots+w_{k}\right)^{2}+\ldots$ in dimension $i$. Of course, in particular, $\bar{w}_{i}$ with $i=n-k+1, n-k+2, \ldots, n$ are the generators of the ideal $I_{n, k}$; see (1). In addition, let $g_{i}\left(w_{2}, \ldots, w_{k}\right)$ (briefly just $g_{i}$ ) denote the reduction of $\bar{w}_{i}\left(w_{1}, \ldots, w_{k}\right)$ modulo $w_{1}$. We note that $\bar{w}_{i}$ is a representative of the Stiefel-Whitney class $w_{i}\left(\gamma^{\perp}\right) \in H^{i}\left(G_{n, k}\right)$; we shall also use $\bar{w}_{i}$ as an abbreviation for $w_{i}\left(\gamma^{\perp}\right)$.

If $i=n-k+1, n-k+2, \ldots, n$, then the polynomials $g_{i}\left(w_{2}, \ldots, w_{k}\right)$ are representatives of some multiples (by an abuse of notation, also denoted by $g_{i}\left(w_{2}, \ldots, w_{k}\right)$, briefly $g_{i}$ ) of the first Stiefel-Whitney class $w_{1}$ in the quotient algebra $H^{*}\left(G_{n, k}\right)$. The singular elements of the standard basis in $H^{n-k+x}\left(G_{n, k}\right)(x \geqslant 0)$, when multiplied by $w_{1}$, do not produce elements of the standard basis in $H^{n-k+x+1}\left(G_{n, k}\right)$. Combined with Lemma 2.3 (where we take those elements of the standard basis divisible by $w_{1}$ in the rôle of the vectors $\vec{a}_{i}$ and the others in the rôle of the vectors $\vec{b}_{j}$ ), this explains why the following proposition focuses on elements of the form $w_{2}^{b_{2}} \cdots w_{k}^{b_{k}} g_{n-k+1+i} \in H^{n-k+x+1}\left(G_{n, k}\right)(i=0,1, \ldots, k-1)$.

Proposition 2.4. For a non-negative integer $x$, we associate with $H^{n-k+x+1}\left(G_{n, k}\right)$ $(2 \leqslant k \leqslant n-k)$ the set

$$
N_{x}\left(G_{n, k}\right):=\bigcup_{i=0}^{k-1}\left\{w_{2}^{b_{2}} \cdots w_{k}^{b_{k}} g_{n-k+1+i} ; 2 b_{2}+3 b_{3}+\cdots+k b_{k}=x-i\right\} .
$$

(1) The cardinality of $N_{x}\left(G_{n, k}\right)$ is equal to $p(\{1,2, \ldots, k-1\}, x)$, which is the same (by Proposition 2.1) as the number of singular elements in the standard basis for $H^{n-k+x}\left(G_{n, k}\right)$.

(2) If $x \leqslant n-k-1$, then each element of $N_{x}\left(G_{n, k}\right)$ consists exclusively of monomials $w_{2}^{c_{2}} \cdots w_{k}^{c_{k}}$ such that $c_{2}+\cdots+c_{k} \leqslant n-k$, thus of elements not divisible by $w_{1}$ and belonging to the standard basis of $H^{n-k+x+1}\left(G_{n, k}\right)$. 
(3) If $x \leqslant n-k-1$ and there are $t$ linearly independent (over $\mathbb{Z}_{2}$ ) elements in the set $N_{x}\left(G_{n, k}\right)$, then

$$
\alpha_{n-k+x}\left(\widetilde{G}_{n, k}\right) \leqslant p(\{1,2, \ldots, k-1\}, x)-t .
$$

In particular, if $x \leqslant n-k-1$ and the set $N_{x}\left(G_{n, k}\right)$ is linearly independent, then

$$
w_{1}: H^{n-k+x}\left(G_{n, k}\right) \longrightarrow H^{n-k+x+1}\left(G_{n, k}\right)
$$

is a monomorphism and $\alpha_{n-k+x}\left(\widetilde{G}_{n, k}\right)$ vanishes.

Proof. Part (1). The set $\left\{w_{2}^{b_{2}} \cdots w_{k}^{b_{k}} g_{n-k+1+i} ; 2 b_{2}+3 b_{3}+\cdots+k b_{k}=x-i\right\}$ consists of $p(\{2, \ldots, k\}, x-i)$ elements. Thus the cardinality of $N_{x}\left(G_{n, k}\right)$ is equal to

$$
\sum_{i=0}^{k-1} p(\{2, \ldots, k\}, x-i) .
$$

If $S$ is a set of positive integers and $p(S, j)$ is the number of partitions of $j$ whose parts are from $S$, then (see [1, Theorem 1.1] if needed) we have, for $|q|<1$,

$$
\sum_{i \geqslant 0} p(S, i) q^{i}=\prod_{i \in S}\left(1-q^{i}\right)^{-1}
$$

Since

$$
\left(1+q+q^{2}+\cdots+q^{k-1}\right) \prod_{i=2}^{k}\left(1-q^{i}\right)^{-1}=\prod_{i=1}^{k-1}\left(1-q^{i}\right)^{-1},
$$

the cardinality of $N_{x}\left(G_{n, k}\right)$ (the sum in $\left.(5)\right)$ is $p(\{1,2, \ldots, k-1\}, x)$.

Part (2). Since each element of $N_{x}\left(G_{n, k}\right)$ is some $w_{2}^{b_{2}} \cdots w_{k}^{b_{k}} g_{n-k+1+i}$ such that $2 b_{2}+3 b_{3}+\cdots+k b_{k}=x-i$, it consists of monomials of the form

$$
w_{2}^{b_{2}} \cdots w_{k}^{b_{k}} w_{2}^{c_{2}} \cdots w_{k}^{c_{k}}=w_{2}^{b_{2}+c_{2}} \cdots w_{k}^{b_{k}+c_{k}},
$$

where $\sum_{i=2}^{k} i\left(b_{i}+c_{i}\right)=n-k+x+1$. Thus, if $x \leqslant n-k-1$, then

$$
\sum_{i=2}^{k}\left(b_{i}+c_{i}\right) \leqslant \frac{n-k+x+1}{2} \leqslant n-k .
$$

Part (3). This is obviously implied by Lemma 2.3 and the first two parts of this proposition.

\section{Results on the characteristic rank and cup-length}

In this section, the tools developed in Section 2 yield new bounds or exact results on the characteristic rank of $\widetilde{\gamma}_{n, k}$ (for odd $n$, also on the characteristic rank of $\widetilde{G}_{n, k}$ ). These lead to obtaining infinitely many new exact values of the cup-length of $\widetilde{G}_{n, 3}$, regarded as likely in Fukaya's conjecture [4, p. 196].

Theorem 3.1. For the oriented Grassmann manifold $\widetilde{G}_{n, k}(4 \leqslant 2 k \leqslant n)$, with the unique integer $t$ such that $2^{t-1}<n \leqslant 2^{t}$, we have the following: 
(1) If $n$ is odd, then $\operatorname{charrank}\left(\widetilde{\gamma}_{n, 2}\right) \geqslant n-2$, and if $n$ is even, then $\operatorname{charrank}\left(\widetilde{\gamma}_{n, 2}\right) \geqslant$ $n-3$.

(2) If $s=1$ or $s=2, r$ is a non-negative integer, and $2^{t-1}+\left\lfloor\frac{s-1}{2}\right\rfloor<n<2^{t}-s-$ 2 , then charrank $\left(\widetilde{\gamma}_{n+r, 3+r}\right) \geqslant n+s-2$. If $3 \leqslant s \leqslant 6$ and $2^{t-1}+\left\lfloor\frac{s-1}{2}\right\rfloor<n<$ $2^{t}-s-2$, then charrank $\left(\widetilde{\gamma}_{n, 3}\right) \geqslant n+s-2$.

In addition, if $n$ is odd, then the replacement of the canonical bundle $\widetilde{\gamma}_{n, k}$ by the corresponding manifold $\widetilde{G}_{n, k}$ gives the corresponding result on charrank $\left(\widetilde{G}_{n, k}\right)$.

Proof. It is known ([8, Theorem 2.1] and the final part of its proof) that if $n$ is odd, then $\operatorname{charrank}\left(\widetilde{\gamma}_{n, k}\right)=\operatorname{charrank}\left(\widetilde{G}_{n, k}\right)$. Thus it suffices to prove Parts (1) and (2).

Part (1). By Remark 2.2, charrank $\left(\widetilde{\gamma}_{n, 2}\right) \geqslant n-3$ for all $n$. If $n$ is odd, then $N_{0}\left(G_{n, 2}\right)$ (see Proposition 2.4 ) only contains $g_{n-1}$. From $\left(1+w_{2}\right)^{-1}=1+w_{2}+w_{2}^{2}+$ $w_{2}^{3}+\cdots$, one sees that $g_{n-1} \neq 0$. Thus, by Proposition $2.4(3), \alpha_{n-2}\left(\widetilde{G}_{n, 2}\right)$ vanishes and we have

$$
\operatorname{charrank}\left(\widetilde{\gamma}_{n, 2}\right) \geqslant n-2 .
$$

Part (2) We shall repeatedly use the fact that, by [8, Lemma 2.3(i)],

$$
g_{i}\left(w_{2}, w_{3}\right)=0 \text { if and only if } i=2^{j}-3 \text { for some } j \geqslant 2 \text {. }
$$

The following lemma (when combined with (6)) will also be useful; cf. each of the four tables that occur in the proof of Theorem 3.1.

Lemma 3.2. For $G_{n, 3}$, let $g_{i}$ denote the same polynomial in $\mathbb{Z}_{2}\left[w_{2}, w_{3}\right]$ as in the rest of this paper.

(a) If $m \neq 4$ is such that $g_{m-1} \neq 0$ and $g_{m} \neq 0$, then $w_{2}^{2} g_{m-1}+w_{3} g_{m} \neq 0$.

(b) If $m \neq 9$ is such that $g_{m-2} \neq 0$ and $g_{m+1} \neq 0$, then $w_{2}^{3} g_{m-2}+w_{3} g_{m+1} \neq 0$.

Proof of the lemma. We know [6], for all $j \geqslant 1$, that

$$
g_{j}=\sum_{\frac{j}{3} \leqslant i \leqslant \frac{j}{2}}\left(\begin{array}{c}
i \\
3 i-j
\end{array}\right) w_{2}^{3 i-j} w_{3}^{j-2 i}
$$

Part (a). Of course, a necessary condition for $w_{2}^{2} g_{m-1}+w_{3} g_{m}=0$ is that

$$
w_{2}^{2} \mid g_{m} \text { and } w_{3} \mid g_{m-1} \text {. }
$$

Writing $m=6 a+b(0 \leqslant b \leqslant 5)$, from $(7)$, one either directly sees that $w_{2}^{2} g_{m-1}+$ $w_{3} g_{m} \neq 0$, or that the divisibility condition (8) is not satisfied.

Indeed, if $b=0$, then $g_{m}$, and if $b=1$, then $g_{m-1}$ is equal to $w_{3}^{2 a}+\cdots+w_{2}^{3 a}$, thus the condition (8) fails for $b=0$, 1. Similarly, if $b=2$, then $g_{m}$, and if $b=3$, then $g_{m-1}$ is equal to $w_{2} w_{3}^{2 a}+\cdots+w_{2}^{3 a+1}$, thus the condition (8) fails for $b=2,3$.

If $b=4$, then we see that the condition (8) is fulfilled. But one calculates that $w_{2}^{2} g_{m-1}+w_{3} g_{m}$ is equal to

$$
a w_{2}^{2} w_{3}^{2 a+1}+\cdots+\alpha w_{2}^{3 a-4} w_{3}^{5}+\beta w_{2}^{3 a-1} w_{3}^{3}+a w_{2}^{3 a+2} w_{3},
$$

where the third last and second last coefficients are abbreviated,

$$
\alpha=\left(\begin{array}{c}
3 a-1 \\
5
\end{array}\right)+\left(\begin{array}{c}
3 a \\
4
\end{array}\right), \quad \beta=\left(\begin{array}{c}
3 a \\
3
\end{array}\right)+\left(\begin{array}{c}
3 a+1 \\
2
\end{array}\right) .
$$

Thus, of course, $w_{2}^{2} g_{m-1}+w_{3} g_{m} \neq 0$ if $a$ is odd. For even $a$, one readily verifies 
that if $a=8 l, 8 l+4$, then $\alpha=1$, and if $a=8 l+2,8 l+6$, then $\beta=1$, thus again $w_{2}^{2} g_{m-1}+w_{3} g_{m} \neq 0$.

Finally, if $b=5$, then $g_{m-1}=\cdots+w_{2}^{3 a+2}$, thus $w_{3} \nmid g_{m-1}$.

Part (b). We proceed similarly as in the proof of Part (a). A necessary condition for $w_{2}^{3} g_{m-2}+w_{3} g_{m+1}=0$ is that

$$
w_{2}^{3} \mid g_{m+1} \text { and } w_{3} \mid g_{m-2} .
$$

Writing $m=6 a+b(0 \leqslant b \leqslant 5)$, one either directly sees, from $(7)$, that $w_{2}^{3} g_{m-2}+$ $w_{3} g_{m+1} \neq 0$, or that the divisibility condition (9) is not satisfied. Indeed, if $b=0$, then $g_{m-2}=g_{6 a-2}=g_{6(a-1)+4}=w_{2}^{3 a-1}+\cdots$, and if $b=4$, then $g_{m-2}=g_{6 a+2}=$ $\cdots+w_{2}^{3 a+1}$, thus $w_{3} \nmid g_{m-2}$; the condition (9) fails for $b=0$, 4. If $b=1$, then $g_{m+1}=$ $g_{6 a+2}=w_{2} w_{3}^{2 a}+\cdots$, if $b=2$, then $g_{m+1}=g_{6 a+3}=w_{3}^{2 a+1}+\cdots$, and if $b=5$, then $g_{m+1}=g_{6(a+1)}=w_{3}^{2 a+2}+\cdots$, thus $w_{2}^{3} \nmid g_{m+1}$; the condition (9) fails for $b=1,2,5$. Finally, let us suppose that $b=3$. Then $g_{m+1}=g_{6 a+4}=(a+1) w_{2}^{2} w_{3}^{2 a}+\cdots+w_{2}^{3 a+2}$, thus $w_{2}^{3} \nmid g_{m+1}$ if $a$ is even. It remains to see what happens for odd $a$. If $a=$ $8 l+1(l \geqslant 1)$, then one calculates that $w_{2}^{3} g_{m-2}+w_{3} g_{m+1}=w_{2}^{3} g_{6 a+1}+w_{3} g_{6 a+4}=$ $\cdots+w_{2}^{3 a-10} w_{3}^{9}+\cdots \neq 0$, if $a=8 l+3$, then one calculates that $w_{2}^{3} g_{m-2}+w_{3} g_{m+1}=$ $\cdots+w_{2}^{3 a-1} w_{3}^{3}+\cdots \neq 0$, if $a=8 l+5$, then we have $w_{2}^{3} g_{m-2}+w_{3} g_{m+1}=\cdots+$ $w_{2}^{3 a-7} w_{3}^{7}+\cdots \neq 0$ and, finally, if $a=8 l+7$, then we have $w_{2}^{3} g_{m-2}+w_{3} g_{m+1}=\cdots+$ $w_{2}^{3 a-1} w_{3}^{3}+\cdots \neq 0$. This proves the lemma.

Now we are ready to verify the claims of Theorem $3.1(2)$.

Case $s=1$. We have $2^{t-1}<n<2^{t}-3$ and assumptions of the theorem imply that $n \geqslant 9$. By [8, Theorem 2.1], we know that charrank $\left(\widetilde{\gamma}_{n+r, 3+r}\right) \geqslant n-2$. One readily calculates (see Proposition 2.4) that

$$
N_{2}\left(G_{n, 3}\right)=\left\{w_{2} g_{n-2}\left(w_{2}, w_{3}\right), g_{n}\left(w_{2}, w_{3}\right)\right\} .
$$

Since (see (6)) $w_{2} g_{n-2} \neq 0, g_{n} \neq 0$ and, since $w_{2} g_{n-2}+g_{n}=w_{3} g_{n-3} \neq 0$, the set $N_{2}\left(G_{n, 3}\right)$ is linearly independent. At the same time,

$$
N_{2}\left(G_{n+r, 3+r}\right)=\left\{w_{2} g_{n-2}\left(w_{2}, w_{3}, \ldots, w_{3+r}\right), g_{n}\left(w_{2}, w_{3}, \ldots, w_{3+r}\right)\right\} .
$$

By iterating the obvious "inclusion" $G_{n, k} \rightarrow G_{n+1, k+1}(D \mapsto D \oplus \mathbb{R})$, we obtain an inclusion

$$
j: G_{n, k} \rightarrow G_{n+r, k+r}
$$

such that, for the pullbacks, we have $j^{*}(\gamma) \cong \gamma \oplus r \varepsilon$ (here $r \varepsilon$ is the trivial $r$-plane bundle) and $j^{*}\left(\gamma^{\perp}\right) \cong \gamma^{\perp}$. Of course, for the induced cohomology homomorphism, we have that $j^{*}\left(w_{i}\right)=w_{i}$ (with the right-hand side zero when $k=3$ and $i \geqslant 4$ ) and $j^{*}\left(\bar{w}_{i}\right)=\bar{w}_{i}$. Thus, since the set $N_{2}\left(G_{n, 3}\right)=j^{*}\left(N_{2}\left(G_{n+r, 3+r}\right)\right)$ is linearly independent, $N_{2}\left(G_{n+r, 3+r}\right)$ has this property as well. Proposition 2.4(3) implies that $\alpha_{n-1}\left(\widetilde{G}_{n+r, 3+r}\right)=0$ and $\operatorname{charrank}\left(\widetilde{\gamma}_{n+r, 3+r}\right) \geqslant n-1$.

Case $s=2$. Now $2^{t-1}<n<2^{t}-4$ and assumptions of the theorem imply that $n \geqslant 9$. By the result for $s=1$, we know that $\operatorname{charrank}\left(\widetilde{\gamma}_{n+r, 3+r}\right) \geqslant n-1$. Since $w_{3} g_{n-2} \neq 0, w_{2} g_{n-1} \neq 0$, and $w_{3} g_{n-2}+w_{2} g_{n-1}=g_{n+1} \neq 0$, the set

$$
N_{3}\left(G_{n, 3}\right)=\left\{w_{3} g_{n-2}, w_{2} g_{n-1}\right\}
$$

is linearly independent. Similarly to the case of $s=1$, one sees for $r>0$ that the set $N_{3}\left(G_{n+r, 3+r}\right)=\left\{w_{3} g_{n-2}, w_{2} g_{n-1}, g_{n+1}\right\}$ is independent. Thus Proposition 2.4(3) 
implies that we have $\alpha_{n}\left(\widetilde{G}_{n+r, 3+r}\right)=0$ and $\operatorname{charrank}\left(\widetilde{\gamma}_{n+r, 3+r}\right) \geqslant n$.

Case $s=3$. We have $2^{t-1}+1<n<2^{t}-5$; assumptions of the theorem imply that $n \geqslant 10$. By the result for $s=2$, we know that $\operatorname{charrank}\left(\widetilde{\gamma}_{n, 3}\right) \geqslant n$. One verifies that $N_{4}\left(G_{n, 3}\right)$ consists precisely of the obviously nonvanishing elements $w_{2}^{2} g_{n-2}, w_{3} g_{n-1}$, and $w_{2} g_{n}$; they are linearly independent, as the following table shows.

\begin{tabular}{|l|l|l|l|}
\hline$h_{1}$ & $h_{2}$ & $h_{3}$ & $h_{1} w_{2}^{2} g_{n-2}+h_{2} w_{3} g_{n-1}+h_{3} w_{2} g_{n}$ \\
\hline \hline 0 & 1 & 1 & $g_{n+2} \neq 0$ \\
\hline 1 & 0 & 1 & $w_{2} w_{3} g_{n-3} \neq 0$ \\
\hline 1 & 1 & 0 & $w_{2}^{2} g_{n-2}+w_{3} g_{n-1} \neq 0$, Lemma 3.2(a) \\
\hline 1 & 1 & 1 & $w_{3}^{2} g_{n-4} \neq 0$ \\
\hline
\end{tabular}

By Proposition $2.4(3)$, now $\alpha_{n+1}\left(\widetilde{G}_{n, 3}\right)=0$ and $\operatorname{charrank}\left(\widetilde{\gamma}_{n, 3}\right) \geqslant n+1$.

Case $s=4$. Now $2^{t-1}+1<n<2^{t}-6$ and, by assumptions of the theorem, we have $n \geqslant 18$. By the result for $s=3$, we know that $\operatorname{charrank}\left(\widetilde{\gamma}_{n, 3}\right) \geqslant n+1$. We see that $N_{5}\left(G_{n, 3}\right)$ consists precisely of the obviously nonvanishing elements $w_{2} w_{3} g_{n-2}$, $w_{2}^{2} g_{n-1}$, and $w_{3} g_{n}$; they are linearly independent, as the following table shows.

\begin{tabular}{|l|l|l|l|}
\hline$h_{1}$ & $h_{2}$ & $h_{3}$ & $h_{1} w_{2} w_{3} g_{n-2}+h_{2} w_{2}^{2} g_{n-1}+h_{3} w_{3} g_{n}$ \\
\hline \hline 0 & 1 & 1 & $w_{2}^{2} g_{n-1}+w_{3} g_{n} \neq 0$, Lemma 3.2(a) \\
\hline 1 & 0 & 1 & $w_{3}^{2} g_{n-3} \neq 0$ \\
\hline 1 & 1 & 0 & $w_{2} g_{n+1} \neq 0$ \\
\hline 1 & 1 & 1 & $g_{n+3} \neq 0$ \\
\hline
\end{tabular}

So we have proved that $\alpha_{n+2}\left(\widetilde{G}_{n, 3}\right)=0$, and Proposition $2.4(3)$ implies that now $\operatorname{charrank}\left(\widetilde{\gamma}_{n, 3}\right) \geqslant n+2$.

Case $s=5$. We have $2^{t-1}+2<n<2^{t}-7$ and assumptions of the theorem imply that $n \geqslant 19$. By the result for $s=4$, we know that $\operatorname{charrank}\left(\widetilde{\gamma}_{n, 3}\right) \geqslant n+2$. One calculates that $N_{6}\left(G_{n, 3}\right)$ consists precisely of the obviously nonvanishing elements $w_{2}^{3} g_{n-2}$, $w_{3}^{2} g_{n-2}, w_{2} w_{3} g_{n-1}$, and $w_{2}^{2} g_{n}$. The following table shows that they are linearly independent.

\begin{tabular}{|l|l|l|l|l|}
\hline$h_{1}$ & $h_{2}$ & $h_{3}$ & $h_{4}$ & $h_{1} w_{2}^{3} g_{n-2}+h_{2} w_{3}^{2} g_{n-2}+h_{3} w_{2} w_{3} g_{n-1}+h_{4} w_{2}^{2} g_{n}$ \\
\hline \hline 0 & 0 & 1 & 1 & $w_{2} g_{n+2} \neq 0$ \\
\hline 0 & 1 & 0 & 1 & $g_{n+4} \neq 0$ \\
\hline 1 & 0 & 0 & 1 & $w_{2}^{2} w_{3} g_{n-3} \neq 0$ \\
\hline 0 & 1 & 1 & 0 & $w_{3} g_{n+1} \neq 0$ \\
\hline 1 & 0 & 1 & 0 & $w_{2}\left(w_{2}^{2} g_{n-2}+w_{3} g_{n-1}\right) \neq 0$, Lemma 3.2(a) \\
\hline 1 & 1 & 0 & 0 & $\left(w_{2}^{3}+w_{3}^{2}\right) g_{n-2} \neq 0$ \\
\hline 0 & 1 & 1 & 1 & $w_{2}^{2} g_{n}+w_{3} g_{n+1} \neq 0$, Lemma 3.2(a) \\
\hline 1 & 0 & 1 & 1 & $w_{2} w_{3}^{2} g_{n-4} \neq 0$ \\
\hline 1 & 1 & 0 & 1 & $w_{3}\left(w_{2}^{2} g_{n-3}+w_{3} g_{n-2}\right) \neq 0$, Lemma $3.2(\mathrm{a})$ \\
\hline 1 & 1 & 1 & 0 & $w_{2}^{3} g_{n-2}+w_{3} g_{n+1} \neq 0$, Lemma 3.2(b) \\
\hline 1 & 1 & 1 & 1 & $w_{3}^{3} g_{n-5} \neq 0$ \\
\hline
\end{tabular}


So we have proved that now $\alpha_{n+3}\left(\widetilde{G}_{n, 3}\right)=0$. By Proposition $2.4(3)$, we have

$$
\operatorname{charrank}\left(\widetilde{\gamma}_{n, 3}\right) \geqslant n+3 .
$$

Case $s=6$. We have $2^{t-1}+2<n<2^{t}-8$ and assumptions of the theorem imply that $n \geqslant 19$. By the result for $s=5$, we know that charrank $\left(\widetilde{\gamma}_{n, 3}\right) \geqslant n+3$. One calculates that $N_{7}\left(G_{n, 3}\right)$ consists precisely of the obviously nonvanishing elements $w_{2}^{2} w_{3} g_{n-2}, w_{2}^{3} g_{n-1}, w_{3}^{2} g_{n-1}$, and $w_{2} w_{3} g_{n}$. The following table shows that they are linearly independent.

\begin{tabular}{|l|l|l|l|l|}
\hline$h_{1}$ & $h_{2}$ & $h_{3}$ & $h_{4}$ & $h_{1} w_{2}^{2} w_{3} g_{n-2}+h_{2} w_{2}^{3} g_{n-1}+h_{3} w_{3}^{2} g_{n-1}+h_{4} w_{2} w_{3} g_{n}$ \\
\hline \hline 0 & 0 & 1 & 1 & $w_{3} g_{n+2} \neq 0$ \\
\hline 0 & 1 & 0 & 1 & $w_{2}\left(w_{2}^{2} g_{n-1}+w_{3} g_{n}\right) \neq 0$, Lemma $3.2(\mathrm{a})$ \\
\hline 1 & 0 & 0 & 1 & $w_{2} w_{3}^{2} g_{n-3} \neq 0$ \\
\hline 0 & 1 & 1 & 0 & $\left(w_{2}^{3}+w_{3}^{2}\right) g_{n-1} \neq 0$ \\
\hline 1 & 0 & 1 & 0 & $w_{3}\left(w_{2}^{2} g_{n-2}+w_{3} g_{n-1}\right) \neq 0$, Lemma $3.2(\mathrm{a})$ \\
\hline 1 & 1 & 0 & 0 & $w_{2}^{2} g_{n+1} \neq 0$ \\
\hline 0 & 1 & 1 & 1 & $w_{2}^{3} g_{n-1}+w_{3} g_{n+2} \neq 0$, Lemma 3.2(b) \\
\hline 1 & 0 & 1 & 1 & $w_{3}^{3} g_{n-4} \neq 0$ \\
\hline 1 & 1 & 0 & 1 & $w_{2} g_{n+3} \neq 0$ \\
\hline 1 & 1 & 1 & 0 & $w_{2}^{2} g_{n+1}+w_{3}^{2} g_{n-1}=g_{n+5} \neq 0$ \\
\hline 1 & 1 & 1 & 1 & $w_{2}^{2} g_{n+1}+w_{3} g_{n+2} \neq 0$, Lemma 3.2(a) \\
\hline
\end{tabular}

So we have proved that now $\alpha_{n+4}\left(\widetilde{G}_{n, 3}\right)=0$. By Proposition $2.4(3)$, we have

$$
\operatorname{charrank}\left(\widetilde{\gamma}_{n, 3}\right) \geqslant n+4 .
$$

The proof of Theorem 3.1 is finished.

Conjecture 3.3. We conjecture that Theorem 3.1(2) holds true for all $s \geqslant 1$ such that $2^{t-1}+\left\lfloor\frac{s-1}{2}\right\rfloor<n<2^{t}-s-2$, and not just when $s \leqslant 6$.

In the proof of Theorem 3.1(2) for $s=1$ or $s=2$, we have extended a specific lower bound for the characteristic rank of $\widetilde{G}_{n, 3}$ to a lower bound for the characteristic rank of $\widetilde{G}_{n+r, 3+r}(r \geqslant 0)$. The following theorem brings an additional piece of information on the homomorphism $w_{1}$ and offers further possibilities for extensions of results on the characteristic rank of the vector bundle $\widetilde{\gamma}_{n, k}$.

Proposition 3.4. For the Grassmann manifold $G_{n, k}(1 \leqslant k \leqslant n-k)$ and any positive integer $l$ not exceeding $n-1$, we have the following:

(1) If the homomorphism $w_{1}: H^{l}\left(G_{n, k}\right) \longrightarrow H^{l+1}\left(G_{n, k}\right)$ is injective, then also $w_{1}: H^{l}\left(G_{n+1, k+1}\right) \longrightarrow H^{l+1}\left(G_{n+1, k+1}\right)$ is injective.

(2) An obvious consequence of (1) is that if $\operatorname{charrank}\left(\widetilde{\gamma}_{n, k}\right) \geqslant l$ then, for any nonnegative integer $r$, we have charrank $\left(\widetilde{\gamma}_{n+r, k+r}\right) \geqslant l$. 
Proof. Let $j: G_{n, k} \rightarrow G_{n+1, k+1}$ denote the inclusion described in (10). The diagram

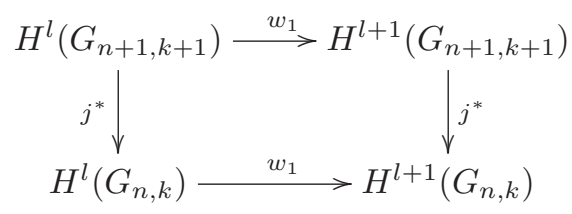

obviously commutes. Let us suppose that the lower homomorphism $w_{1}$ is injective; we should prove that the upper homomorphism $w_{1}$ is injective as well.

The standard basis vectors (see (4)) in $H^{l}\left(G_{n+1, k+1}\right)$ are

$$
w_{1}^{a_{1}}\left(\gamma_{n+1, k+1}\right) \cdots w_{k}^{a_{k}}\left(\gamma_{n+1, k+1}\right) w_{k+1}^{a_{k+1}}\left(\gamma_{n+1, k+1}\right)
$$

such that $a_{1}+2 a_{2}+\cdots+k a_{k}+(k+1) a_{k+1}=l$ and $a_{1}+a_{2}+\cdots+a_{k}+a_{k+1} \leqslant$ $n-k$. The images, under $w_{1}: H^{l}\left(G_{n+1, k+1}\right) \rightarrow H^{l+1}\left(G_{n+1, k+1}\right)$, of those vectors (11) having $a_{k+1}=0$ are linearly independent. Indeed, these images are

$$
w_{1}^{1+a_{1}}\left(\gamma_{n+1, k+1}\right) \cdots w_{k}^{a_{k}}\left(\gamma_{n+1, k+1}\right) ;
$$

the vectors $j^{*}\left(w_{1}^{1+a_{1}}\left(\gamma_{n+1, k+1}\right) \cdots w_{k}^{a_{k}}\left(\gamma_{n+1, k+1}\right)\right)=w_{1}^{1+a_{1}}\left(\gamma_{n, k}\right) \cdots w_{k}^{a_{k}}\left(\gamma_{n, k}\right)$ (being images of the standard basis vectors $w_{1}^{a_{1}}\left(\gamma_{n, k}\right) \cdots w_{k}^{a_{k}}\left(\gamma_{n, k}\right) \in H^{l}\left(G_{n, k}\right)$ under the injective linear map $\left.w_{1}: H^{l}\left(G_{n, k}\right) \rightarrow H^{l}\left(G_{n, k}\right)\right)$ are linearly independent. Thus also $w_{1}^{1+a_{1}}\left(\gamma_{n+1, k+1}\right) \cdots w_{k}^{a_{k}}\left(\gamma_{n+1, k+1}\right)$ are linearly independent. In addition, the images under $w_{1}: H^{l}\left(G_{n+1, k+1}\right) \rightarrow H^{l+1}\left(G_{n+1, k+1}\right)$ of those vectors (11) having $a_{k+1} \geqslant 1$ are also linearly independent, because all the standard basis vectors (11) having $a_{k+1} \geqslant 1$ are regular. Indeed, we have for any of these standard basis vectors in $H^{l}\left(G_{n+1, k+1}\right)$ that

$$
\left(a_{1}+a_{2}+\cdots+a_{k}+a_{k+1}\right)+k a_{k+1} \leqslant a_{1}+2 a_{2}+\cdots+k a_{k}+(k+1) a_{k+1}=l,
$$

thus

$$
a_{1}+a_{2}+\cdots+a_{k}+a_{k+1} \leqslant l-k a_{k+1} \leqslant l-k \leqslant n-k-1 .
$$

Finally, the $w_{1}$-images of all the standard basis vectors (11) of $H^{l}\left(G_{n+1, k+1}\right)$ are linearly independent. Indeed, let us suppose that a linear combination of all these images vanishes, that is,

$$
\sum \alpha_{\left(a_{1}, \ldots, a_{k}, 0\right)} w_{1}^{1+a_{1}} w_{2}^{a_{2}} \cdots w_{k}^{a_{k}}+\sum_{a_{k+1} \geqslant 1} \alpha_{\left(a_{1}, \ldots, a_{k}, a_{k+1}\right)} w_{1}^{1+a_{1}} w_{2}^{a_{2}} \cdots w_{k}^{a_{k}} w_{k+1}^{a_{k+1}}=0 .
$$

When mapped by $j^{*}: H^{l+1}\left(G_{n+1, k+1}\right) \rightarrow H^{l+1}\left(G_{n, k}\right)$, this gives that

$$
\sum \alpha_{\left(a_{1}, \ldots, a_{k}, 0\right)} w_{1}^{1+a_{1}}\left(\gamma_{n, k}\right) w_{2}^{a_{2}}\left(\gamma_{n, k}\right) \cdots w_{k}^{a_{k}}\left(\gamma_{n, k}\right)=0
$$

implying that all the coefficients $\alpha_{\left(a_{1}, \ldots, a_{k}, 0\right)}$ vanish, since $w_{1}^{1+a_{1}}\left(\gamma_{n, k}\right) \cdots w_{k}^{a_{k}}\left(\gamma_{n, k}\right)$ are linearly independent. So the left-hand side of (12) is reduced to a linear combination of vectors already known to be linearly independent, we have

$$
\sum_{a_{k+1} \geqslant 1} \alpha_{\left(a_{1}, \ldots, a_{k}, a_{k+1}\right)} w_{1}^{1+a_{1}}\left(\gamma_{n+1, k+1}\right) w_{2}^{a_{2}}\left(\gamma_{n+1, k+1}\right) \cdots w_{k+1}^{a_{k+1}}\left(\gamma_{n+1, k+1}\right)=0,
$$

thus also all the coefficients $\alpha_{\left(a_{1}, \ldots, a_{k}, a_{k+1}\right)}\left(a_{k+1} \geqslant 1\right)$ must vanish. This finishes the proof of Proposition 3.4. 
Remark 3.5. The assumption $l \leqslant n-1$ in Proposition 3.4(1) is the best possible, in the sense that the claim is false, in general, for $l=n$. Indeed, $w_{1}: H^{7}\left(G_{7,2}\right) \rightarrow$ $H^{8}\left(G_{7,2}\right)$ is readily seen to be a monomorphism (apply Proposition 2.4(3); the set $N_{2}\left(G_{7,2}\right)=\left\{w_{2} g_{6}\right\}$ is linearly independent), but the homomorphism $w_{1}: H^{7}\left(G_{8,3}\right) \rightarrow$ $H^{8}\left(G_{8,3}\right)$ is not injective (by a calculation in the cohomology algebra $H^{*}\left(G_{8,3}\right),(1)$, or consulting Stong's result on the height of $w_{1}$ in [13], one sees that the kernel of this homomorphism contains $w_{1}^{7} \neq 0$ ).

Theorem 3.1 enables us to derive, among others, new exact results on the characteristic rank and $\mathbb{Z}_{2}$-cup-length of three infinite families of the manifolds $\widetilde{G}_{n, 3}$.

Theorem 3.6. For the oriented Grassmann manifolds $\widetilde{G}_{n, k}(4 \leqslant 2 k \leqslant n)$ we have the following:

(1) If $n$ is odd, then

$$
\operatorname{charrank}\left(\widetilde{\gamma}_{n, 2}\right)=n-2, \operatorname{cup}\left(\widetilde{G}_{n, 2}\right)=\frac{n-1}{2},
$$

and if $n$ is even, then

$$
\operatorname{charrank}\left(\widetilde{\gamma}_{n, 2}\right)=n-3, \operatorname{cup}\left(\widetilde{G}_{n, 2}\right)=\frac{n}{2} .
$$

(2) If $q \geqslant 4$, then

$$
\begin{gathered}
\operatorname{charrank}\left(\widetilde{\gamma}_{2^{q-1}+1,3}\right)=2^{q-1}+1, \operatorname{cup}\left(\widetilde{G}_{2^{q-1}+1,3}\right)=2^{q-1}-3, \\
\operatorname{charrank}\left(\widetilde{\gamma}_{10,3}\right)=11, \operatorname{cup}\left(\widetilde{G}_{10,3}\right)=5,
\end{gathered}
$$

and, if $q \geqslant 5$, then

$$
\begin{aligned}
& \operatorname{charrank}\left(\widetilde{\gamma}_{2^{q-1}+2,3}\right)=2^{q-1}+4, \operatorname{cup}\left(\widetilde{G}_{2^{q-1}+2,3}\right)=2^{q-1}-3, \\
& \operatorname{charrank}\left(\widetilde{\gamma}_{2^{q-1}+3,3}\right)=2^{q-1}+7, \operatorname{cup}\left(\widetilde{G}_{2^{q-1}+3,3}\right)=2^{q-1}-3 .
\end{aligned}
$$

Remark 3.7. The results on the cup-length in Theorem 3.6(2) confirm the corresponding claims of Fukaya's conjecture [4, p. 196]; another claim contained in this conjecture was proved in $[\mathbf{8}]$.

Proof. Part (1). Let us first suppose that $n$ is odd. It is clear (for instance, from (1)) that $w_{2}^{\frac{n-3}{2}} \in H^{n-3}\left(G_{n, 2}\right)$ is not a multiple of $w_{1}$, thus we have $\widetilde{w}_{2}^{\frac{n-3}{2}} \neq 0$ and $\operatorname{cup}\left(\widetilde{G}_{n, 2}\right) \geqslant \frac{n-1}{2}$. We know, from Theorem 3.1, that $\operatorname{charrank}\left(\widetilde{\gamma}_{n, 2}\right) \geqslant n-2$. Thus Theorem 1.1 implies that $\operatorname{cup}\left(\widetilde{G}_{n, 2}\right) \leqslant \frac{n-1}{2}$, and we see that $\operatorname{cup}\left(\widetilde{G}_{n, 2}\right)=\frac{n-1}{2}$, as claimed. At the same time, this shows that $\operatorname{charrank}\left(\widetilde{\gamma}_{n, 2}\right) \leqslant n-2\left(\operatorname{charrank}\left(\widetilde{\gamma}_{n, 2}\right) \geqslant\right.$ $n-1$ would imply a false inequality, $\left.\operatorname{cup}\left(\widetilde{G}_{n, 2}\right) \leqslant \frac{n-2}{2}\right)$, and so $\operatorname{charrank}\left(\widetilde{\gamma}_{n, 2}\right)=$ $n-2$. [To see that charrank $\left(\widetilde{\gamma}_{n, 2}\right) \leqslant n-2$, it also suffices to compare the Betti numbers $b_{n-1}\left(G_{n, 2}\right)=\frac{n-1}{2}$ and $b_{n}\left(G_{n, 2}\right)=\frac{n-3}{2}$, readily calculated from the Poincaré polynomial.]

Now let us suppose that $n$ is even. First, we note that $\widetilde{G}_{4,2} \cong S^{2} \times S^{2}$; clearly $\chi_{2}\left(\widetilde{G}_{4,2}\right)=1=\alpha_{2}\left(\widetilde{G}_{4,2}\right)$, $\operatorname{charrank}\left(\widetilde{\gamma}_{4,2}\right)=1$, and $\operatorname{cup}\left(\widetilde{G}_{4,2}\right)=2$, as claimed. So we may suppose that $n \geqslant 6$. Then $w_{2}^{\frac{n-2}{2}} \in H^{n-2}\left(G_{n, 2}\right)$ cannot be a multiple of $w_{1}$, thus 
we have $\widetilde{w}_{2}^{\frac{n-2}{2}} \neq 0$ and $\operatorname{cup}\left(\widetilde{G}_{n, 2}\right) \geqslant \frac{n}{2}$. We know, from Theorem 3.1, that

$$
\operatorname{charrank}\left(\widetilde{\gamma}_{n, 2}\right) \geqslant n-3
$$

Theorem 1.1 gives $\operatorname{cup}\left(\widetilde{G}_{n, 2}\right)=\frac{n}{2}$. We know, from Theorem 3.1, that

$$
\operatorname{charrank}\left(\widetilde{\gamma}_{n, 2}\right) \geqslant n-3 .
$$

Admitting that $\operatorname{charrank}\left(\widetilde{\gamma}_{n, 2}\right) \geqslant n-2$ implies a false inequality, $\operatorname{cup}\left(\widetilde{G}_{n, 2}\right) \leqslant \frac{n-1}{2}$. [An alternative: since $b_{n-2}\left(G_{n, 2}\right)=\frac{n}{2}$ and $b_{n-1}\left(G_{n, 2}\right)=\frac{n-3}{2}$, the homomorphism $w_{1}: H^{n-2}\left(G_{n, 2}\right) \longrightarrow H^{n-1}\left(G_{n, 2}\right)$ is not injective, and we conclude that $\operatorname{charrank}\left(\widetilde{\gamma}_{n, 2}\right) \leqslant n-3$.] Thus charrank $\left(\widetilde{\gamma}_{n, 2}\right)=n-3$, as claimed.

Part (2). We first note that, for any non-negative integer $x$, one has an obvious "inclusion" $\tilde{j}: \widetilde{G}_{2^{q-1}, 3} \rightarrow \widetilde{G}_{2^{q-1}+x, 3}$, such that $\tilde{j}^{*}\left(\widetilde{\gamma}_{2^{q-1}+x, 3}\right) \cong \widetilde{\gamma}_{2^{q-1}, 3}$. Thus, in cohomology, $\tilde{j}^{*}\left(w_{2}^{2^{q-1}-4}\left(\widetilde{\gamma}_{2^{q-1}+x, 3}\right)\right)=w_{2}^{2^{q-1}-4}\left(\widetilde{\gamma}_{2^{q-1}, 3}\right)$. It was proved in [7, p. 77$]$ that the latter cohomology class does not vanish. As a consequence, we have that

$$
\operatorname{cup}\left(\widetilde{G}_{2^{q-1}+x, 3}\right) \geqslant 2^{q-1}-3 .
$$

For $\widetilde{G}_{2^{q-1}+1,3}(q \geqslant 4)$, Theorem $3.1(2)$ with $s=2$ implies that

$$
\operatorname{charrank}\left(\widetilde{\gamma}_{2^{q-1}+1,3}\right) \geqslant 2^{q-1}+1 .
$$

Then, from Theorem 1.1, we obtain that $\operatorname{cup}\left(\widetilde{G}_{2^{q-1}+1,3}\right) \leqslant 2^{q-1}-3$, thus we have $($ see $(13)) \operatorname{cup}\left(\widetilde{G}_{2^{q-1}+1,3}\right)=2^{q-1}-3$ and $\operatorname{charrank}\left(\widetilde{\gamma}_{2^{q-1}+1,3}\right)=2^{q-1}+1$.

For $\widetilde{G}_{2^{q-1}+2,3}$ with $q=4$, that is, for $\widetilde{G}_{10,3}$, Theorem $3.1(2)$ with $s=3$ applies and gives that $\operatorname{charrank}\left(\widetilde{\gamma}_{10,3}\right)=11$. Thus from Theorem 1.1 , we obtain that $\operatorname{cup}\left(\widetilde{G}_{10,3}\right) \leqslant$ 5 which, when combined with (13), implies that $\operatorname{cup}\left(\widetilde{G}_{10,3}\right)=5$ and $\operatorname{charrank}\left(\widetilde{\gamma}_{10,3}\right)=$ 11. Let us continue with $\widetilde{G}_{2^{q-1}+2,3}, q \geqslant 5$. Then Theorem 3.1(2) with $s=4$ implies that $\operatorname{charrank}\left(\widetilde{\gamma}_{2^{q-1}+2,3}\right) \geqslant 2^{q-1}+4$. From Theorem 1.1, we see that $\operatorname{cup}\left(\widetilde{G}_{2^{q-1}+2,3}\right) \leqslant$ $2^{q-1}-3$; this, jointly with (13), yields

$$
\operatorname{cup}\left(\widetilde{G}_{2^{q-1}+2,3}\right)=2^{q-1}-3 \text { and } \operatorname{charrank}\left(\widetilde{\gamma}_{2^{q-1}+2,3}\right)=2^{q-1}+4,
$$

as claimed.

For $\widetilde{G}_{2^{q-1}+3,3}$ with $q \geqslant 5$, we apply Theorem 3.1(2) with $s=6$ and see that $\operatorname{charrank}\left(\widetilde{\gamma}_{2^{q-1}+3,3}\right) \geqslant 2^{q-1}+7$. Theorem 1.1 implies that $\operatorname{cup}\left(\widetilde{G}_{2^{q-1}+3,3}\right) \leqslant 2^{q-1}-3$ which, when combined with (13), shows that

$$
\operatorname{cup}\left(\widetilde{G}_{2^{q-1}+3,3}\right)=2^{q-1}-3 \text { and } \operatorname{charrank}\left(\widetilde{\gamma}_{2^{q-1}+3,3}\right)=2^{q-1}+7,
$$

indeed. The proof of Theorem 3.6 is finished.

\section{Acknowledgments}

The authors thank Professor Peter Zvengrowski and the referee for useful comments related to the presentation of this paper. 


\section{References}

[1] G. Andrews, The Theory of Partitions, Encyclopedia Math. Appl., Vol. 2, Addison-Wesley, Reading, MA, 1976.

[2] L'. Balko and J. Korbaš, A note on the characteristic rank of null-cobordant manifolds, Acta Math. Hungar. 140 (2013), 145-150.

[3] A. Borel, La cohomologie mod 2 de certains espaces homogènes, Comment. Math. Helv. 27 (1953), 165-197.

[4] T. Fukaya, Gröbner bases of oriented Grassmann manifolds, Homology, Homotopy Appl. 10 (2008), 195-209.

[5] J. Jaworowski, An additive basis for the cohomology of real Grassmannians, Algebraic Topology Poznań 1989, 231-234, Lecture Notes in Math., Vol. 1474, Springer, Berlin, 1991.

[6] J. Korbaš, Bounds for the cup-length of Poincaré spaces and their applications, Topology Appl. 153 (2006), 2976-2986.

[7] J. Korbaš, The cup-length of the oriented Grassmannians vs a new bound for zero-cobordant manifolds, Bull. Belg. Math. Soc. Simon Stevin 17 (2010), 69-81.

[8] J. Korbaš, The characteristic rank and cup-length in oriented Grassmann manifolds, Osaka J. Math. 52 (2015), 1163-1172.

[9] J. Milnor and J. Stasheff, Characteristic Classes, Princeton University Press, Princeton, NJ; University of Tokyo Press, Tokyo, 1974.

[10] A.C. Naolekar and A.S. Thakur, Note on the characteristic rank of vector bundles, Math. Slovaca 64 (2014), 1525-1540.

[11] I. Schur, Vorlesungen über Invariantentheorie. (German) Bearbeitet und herausgegeben von H. Grunsky. Springer-Verlag, Berlin, New York, 1968.

[12] E.H. Spanier, Algebraic Topology, corrected reprint, Springer-Verlag, New York, Berlin, 1981.

[13] R.E. Stong, Cup products in Grassmannians, Topology Appl. 13 (1982), 103113.

[14] R.E. Stong, Semicharacteristics of oriented Grassmannians, J. Pure Appl. Algebra 33 (1984), 97-103.

Július Korbaš korbas@fmph.uniba.sk

Tomáš Rusin svmydlo@gmail.com

Department of Algebra, Geometry, and Mathematical Education, Faculty of Mathematics, Physics, and Informatics, Comenius University Bratislava, Mlynská dolina, SK-842 48 Bratislava 4, Slovakia 